\title{
Not perfect, but good enough
}

\author{
Gerald Brock, MD, FRCSC
}

See related article on page 187

The authors of this trial ${ }^{1}$ are to be commended for evaluating an important clinical question relating to the reliability and first-dose efficacy of vardenafil in a large, placebo-controlled cohort of men with moderateto-severe erectile dysfunction. This study provided evidence of both excellent reliability (the ability of the drug to consistently enhance erectile function time after time in an individual) and first-dose efficacy. Reading this report, one is struck quickly by a near $80 \%$ Sexual Encounter Profile question 3 (SEP3) result (the ability to get and keep the erection until the end of the sexual event) during the 12-week phase in a group of men with significant rates of dyslipidemia, diabetes and hypertension.

To the casual observer, these values may seem surprisingly high, compared with other large, multicentre, placebo-controlled trials involving any of the phosphodiesterase type 5 (PDE5) inhibitors. In fact, when one looks at the study design, where only responders were entered into the 12-week placebo phase of the study, these results seem more in keeping with other trials. In addition, close to $80 \%$ of the men evaluated in this trial had previously used sildenafil, with $9 \%$ having previously tried tadalafil. It is unclear to me from this report whether nonresponse to other PDE5 inhibitors was an exclusion to entry into this study. If it was, these men would all be PDE5 inhibitor responders, and as such, the men evaluated in this report would represent a significantly enriched population. A more robust study design would clearly have evaluated PDE5 inhibitor-naive patients.

Finally, the use of SEP question 2 (SEP2) as the primary outcome measure should be questioned. SEP2 (the ability to penetrate your partner) is, in my opinion, often positive among men who, although able to penetrate, cannot complete the sex act, lose their rigidity before orgasm and frequently express dissatisfaction with the drug. In essence, a positive SEP2 result means little clinically. Importantly, however, the authors provide robust secondary end points that demonstrate strong efficacy throughout the 12-week study period, using SEP3 and reliability measures that are clearly superior to the placebo arm.

Is this a perfect study? No. It included vardenafil-naive patients but allowed men who had tried the other PDE5 inhibitors. It chose a weak primary outcome measure and studied what appears to be a responder population. Does that mean we learn nothing from it? No. This study, in support of an earlier publication, RELY $1,{ }^{2}$ comprises part of a vast and growing literature that supports PDE5 inhibitors as safe and effective agents for men with erectile dysfunction. First-dose efficacy is an important attribute, as is reliability for the men who use these drugs, and for their partners. I generally tell my patients they're likely to have a success rate of $70 \%-80 \%$, meaning that $7-8$ of 10 men will respond on the first attempt and on 7-8 out of 10 subsequent attempts. This study says I am not far off for vardenafil, using $20 \mathrm{mg}$ in this population of men with significant comorbid conditions.

Professor of Surgery, Division of Urology, University of Western Ontario, London, Ont.

This article has been peer reviewed.

Competing interests: None declared.

\section{References}

1. Valiquette L, Montorsi F, Auerbach S; the Vardenafil Study Group. Vardenafil demonstrates first-dose success and reliability of penetration and maintenance of erection in men with erectile dysfunction - RELY-II. CUAJ 2008;2: 187-95.

2. Valiquette L, Montorsi F, Auerbach S; the Vardenafil Study Group. First-dose success with vardenafil in men with erectile dysfunction and associated comorbidities: RELYII. Int I Clin Pract 2006;60:1378-85.

Correspondence: Dr. Gerald Brock, Professor of Surgery, Division of Urology, University of Western Ontario, 268 Grosvenor St., London ON N6A 4V2; gebrock@sympatico.ca 